\title{
Dynamiques économiques et renouvellement urbain en Roumanie : l'exemple de la ville de Timişoara
}

Economic development and urbain regeneration in Romania. The example of the city of Timisoara

Wirtschaftsdynamik und städtische Neuerung in Rumänien am Beispiel der Stadt Timisoara

Vincent Veschambre et Nicolae Popa

\section{(2) OpenEdition}

Édition électronique

URL : https://journals.openedition.org/rge/895

DOI : $10.4000 /$ rge.895

ISSN : 2108-6478

Éditeur

Association des géographes de l'Est

Édition imprimée

Date de publication : 1 janvier 2009

ISSN : 0035-3213

\section{Référence électronique}

Vincent Veschambre et Nicolae Popa, «Dynamiques économiques et renouvellement urbain en

Roumanie : l'exemple de la ville de Timişoara », Revue Géographique de l'Est [En ligne], vol. 49 / 1 | 2009, mis en ligne le 28 mai 2009, consulté le 21 septembre 2021. URL : http://journals.openedition.org/rge/ 895 ; DOI : https://doi.org/10.4000/rge.895

Ce document a été généré automatiquement le 21 septembre 2021.

Tous droits réservés 


\section{Dynamiques économiques et renouvellement urbain en Roumanie : l'exemple de la ville de Timişoara}

Economic development and urbain regeneration in Romania. The example of the city of Timisoara

Wirtschaftsdynamik und städtische Neuerung in Rumänien am Beispiel der

Stadt Timisoara

Vincent Veschambre et Nicolae Popa

\section{Introduction}

1 Dans une logique de mise en concurrence, l'économie héritée de la période communiste a fait preuve de faibles capacités d'adaptation aux mécanismes du marché. Par conséquent, la plupart des unités industrielles ont réduit leur production ou ont été fermées. L'agriculture s'est orientée vers les pratiques d'autosubsistance, pendant que les services se sont développés inégalement, se concentrant dans les grandes villes. Ces évolutions sont dramatiques dans le milieu rural, mais elles caractérisent aussi l'espace urbain, dont le poids au niveau national a significativement diminué. Timisoara fait partie des quelques villes roumaines qui ont attiré des investissements importants dans le secteur industriel et dans celui des services à forte valeur ajoutée (Popa, 2004).

Tableau 1 : Dynamique des investissements directs étrangers à Timisoara

\begin{tabular}{|l|l|l|l|l|l|l|l|l|}
\hline Année & $1990-2001$ & 2002 & 2003 & 2004 & 2005 & 2006 & 2007 & Total \\
\hline
\end{tabular}




\begin{tabular}{|r|r|r|r|r|r|r|r|r|}
\hline Millions euros & 211,7 & 129,80 & 1,76 & 70,1 & 8,9 & 4,3 & 6,7 & 433,26 \\
\hline
\end{tabular}

(Source : Mairie de Timisoara, 2008)

2 Les investissements directs étrangers les plus importants ont été réalisés avant 2003, quand sont venues s'installer des compagnies européennes, états-uniennes de grande taille comme Alcatel, Siemens, Luxten Lighting (en électronique), Procter\& Gamble (en chimie) etc., à côté d'un grand nombre d'entreprises plus petites, notamment du secteur textiles-habillement et chaussures, puis du commerce. Ensuite, la ville a continué à attirer des investisseurs étrangers, mais de plus petite taille. ${ }^{1} \mathrm{Ce}$ qui a permis au budget de la ville de Timisoara d'être multiplié pratiquement par 5 entre 2000 et 2008. Même si dernièrement son rythme de croissance a été dépassé par celui d'autres villes, comme Cluj et Arad, Timisoara continue d'être en 2008 la deuxième ville de Roumanie par son budget local, après la capitale, notamment grâce a la croissance du secteur tertiaire.

Tableau 2 : Dynamique des revenus budgetaires de la municipalité de Timisoara (\%)

\begin{tabular}{|l|l|l|l|l|l|l|l|l|}
\hline Année & 2000 & 2002 & 2003 & 2004 & 2005 & 2006 & 2007 & 2008 \\
\hline Croissance \% & 100 & 175,7 & 241,3 & 274,8 & 276,9 & 387,1 & 417,1 & 514,4 \\
\hline
\end{tabular}

(Source : Mairie de Timisoara, 2008)

3 Dans quelle mesure la communauté urbaine locale bénéficie-t-elle de cette attractivité économique ? Nous envisagerons ces retombées du point de vue des transformations urbaines, tissu industriel et tertiaire, infrastructures, habitat, mise en valeur des héritages urbains... Ce que nous désignerons sous le terme générique de renouvellement urbain avec cette problématique qui est spécifique aux villes de l'Europe anciennement «socialiste». Dans ces villes, c'est en effet l'ensemble des équipements, des infrastructures, du parc de logement qui était obsolète au moment du changement de régime politique et de système économique: problèmes d'entretien, de mauvaise qualité des constructions... C'est donc le tissu urbain dans son ensemble qui est potentiellement concerné par un tel renouvellement urbain, même si de fait les inerties sont nombreuses.

4 Est-ce que l'ampleur des investissements réalisés à Timisoara induit un rythme de renouvellement urbain particulièrement élevé dans le contexte roumain et conforme à ses ambitions de métropole régionale, voire transfrontalière ? Inversement, les interventions sur le bâti, les espaces publics, le paysage urbain peuvent-elles constituer un facteur d'attractivité supplémentaire pour les investisseurs?

5 Avant de fournir des éléments de réponse, il est nécessaire de présenter succinctement la ville de Timisoara à travers les principaux atouts dont elle dispose pour attirer les investisseurs. La ville occupe en effet la deuxième place, après Bucarest, du point de vue de l'importance des investissements étrangers, avec environ 6500 entreprises étrangères, ce qui représente un tiers de l'emploi enregistré dans la ville.

6 Parmi les points forts qui expliquent une telle attractivité, il faut mentionner tout d'abord la situation privilégiée de la ville. Située à $570 \mathrm{~km}$ de Bucarest, la ville de 
Timisoara est le plus grand centre urbain de l'ouest du pays, sur lequel elle exerce un rayonnement important, possédant la zone d'influence directe la plus large $\left(5000 \mathrm{~km}^{2}\right)$, après la capitale. Par ses fonctions complexes, la ville polarise un territoire beaucoup plus étendu, tout en étant le plus grand centre économique, commercial, culturel et scientifique de la Région de développement Ouest de la Roumanie (Dobraca, 1997, Voiculescu, 2004). Réputée dès la deuxième moitié du XVIIIe siècle pour l'esprit mercantiliste de ses habitants, Timisoara a bénéficié pendant les dernières décennies de régime communiste, du statut de principal point de contact avec «le monde libre ", ce qui lui a conféré un certain cosmopolitisme, une plus grande ouverture au reste du monde. Ce contexte culturel plus favorable à l'initiative individuelle que dans le reste du pays a constitué un atout après la chute du régime de Ceausescu².

7 Bien située à l'intérieur d'un réseau urbain intensément polarisée ${ }^{3}$, Timisoara bénéficie aujourd'hui d'une position favorable, même si le voisinage avec la Serbie demeure à ce jour un handicap (Popa, Junie, 2000, Bioteau, 2005). La ville est à mi-distance entre deux grands pôles urbains de l'Europe centrale et de Sud-Est, Bucarest et Budapest. Elle constitue un point d'appui sur l'axe qui relie le centre-nord de la Méditerranée à l'isthme ponto-baltique. Cette fonction a permis à l'aéroport international de Timisoara de s'affirmer comme la deuxième porte aérienne du pays, après Bucarest. La ville est desservie par nombreuses compagnies aériennes qui la relient à 30 grandes villes européennes, principalement italiennes. Cette histoire et cette position géographique centre-européennes, ont permis à la ville de Timisoara de s'affirmer comme un centre novateur, ouvert sur l'Europe occidentale.

8 Bénéficiant par ailleurs du statut de pôle régional de développement, Timisoara est prioritaire pour les investissements de rang international en matière d'infrastructures, de sites de production et de services. C'est un grand centre universitaire, dans lequel les spécialisations techniques sont traditionnelles et restent dominantes, facilitant ainsi une bonne relation entre la formation supérieure, la recherche, le milieu économique et celui des affaires.

9 L'attractivité de la ville repose également sur les coûts encore relativement bas du foncier, de l'immobilier et de la main d'œuvre. Le revers de la médaille, c'est un déficit de main d'œuvre, accentué par le départ des jeunes et des spécialistes à l'étranger, en quête de meilleurs salaires.

10 Enfin, la présence dans la ville de plusieurs quartiers historiques et de nombreux espaces verts constitue d'ores et déjà une carte de visite appréciable et représente un potentiel de développement. L'organisation de l'espace urbain est le résultat de la reconstruction intégrale réalisée après la défaite des Turcs et la reconquête du Banat par les Habsbourgs à partir de 1716. Le plan radioconcentrique de la Cité (Cetate) (carte $n^{\circ} 1$ ) revêt un intérêt majeur au niveau national, voire européen car il gardé sa structure originelle (Maggi, 2002). 
Carte 1 : Morphologie urbaine du centre ville de Timisoara (Cetate)

Carte $n^{\circ} 1$ : Morphologie urbaine du centre ville de Timisoara (Cetate)

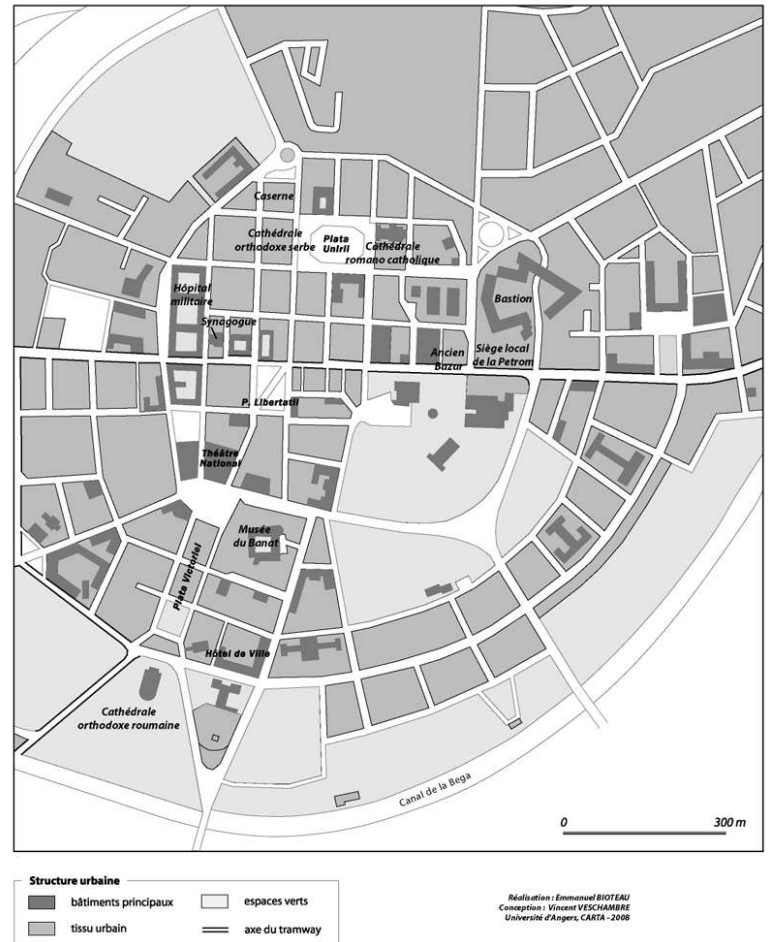

11 C'est cette conservation du plan de la reconstruction au XVIIIème siècle, ainsi que la superposition des époques architecturales (baroque, XIXème, art nouveau...) qui fait l'intérêt et l'originalité de la ville de Timisoara ${ }^{4}$. Le noyau de quartiers anciens péricentraux est également bien lisible, notamment ceux des quartiers Fabric et Josefin $\left(\right.$ carte $\left.n^{\circ} 2\right)$. Cette structure radioconcentrique a orienté les extensions ultérieures, notamment pour la construction de grands immeubles (blocuri), après 1950. Un autre élément qui confère une forte personnalité à la ville est le chapelet de parcs et d'espaces verts qui longe la Bega, rivière anciennement canalisée qui traverse la ville, d'Est en Ouest, au sud du quartier central (Cetate). 
Carte 2 : Espaces patrimonialisés et renouvellement urbain dans les quartiers centraux et péricentraux de Timisoara

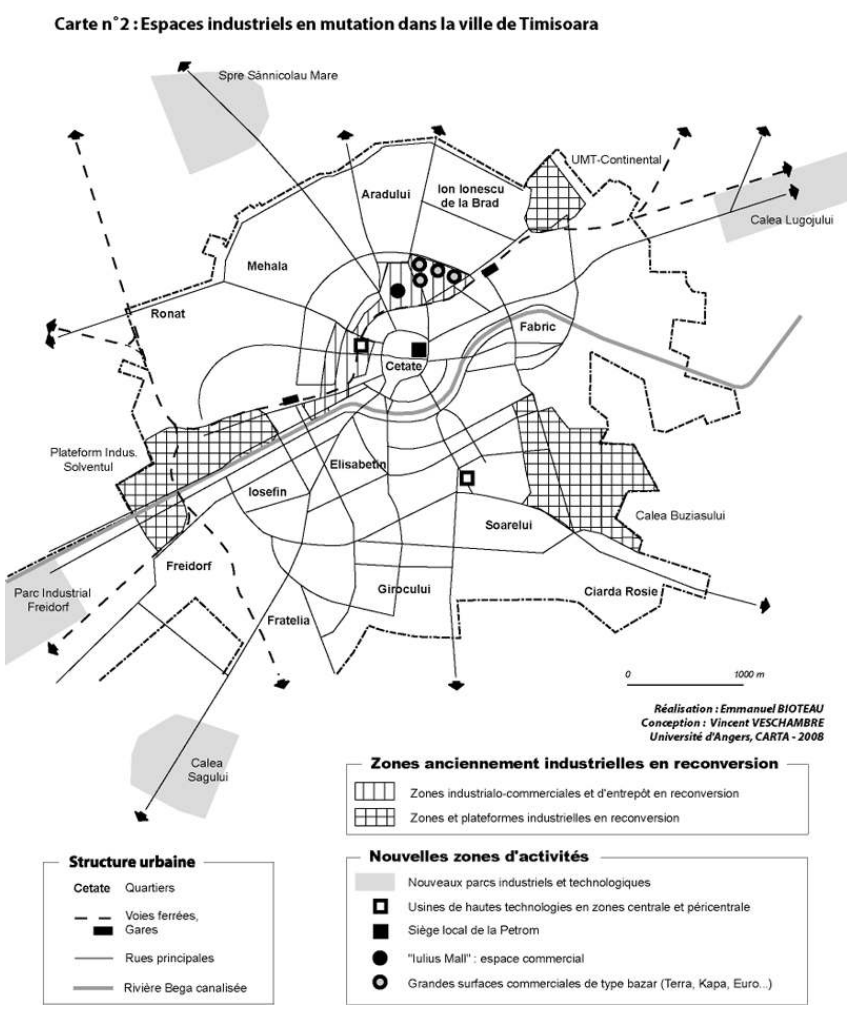

12 C'est à partir des ces héritages patrimoniaux mais aussi d'héritages industriels plus difficiles à valoriser que s'est engagé à Timisoara un processus de recyclage du tissu urbain qui va en s'accélérant depuis quelques années. Dans le même temps sont apparus de nouveaux espaces d'activité et d'habitat individuel en périphérie, ce qui est tout à fait nouveau pour une ville roumaine. Nous ne traiterons ici que du premier volet de ces transformations urbaines, celles qui relèvent à proprement parler du renouvellement urbain. Les investisseurs privés jouent dans ce domaine un rôle important mais encore limité. L'engagement des autorités locales apparaît décisif pour dynamiser et orienter ce renouvellement urbain, autour notamment de certains éléments forts du patrimoine urbain.

\section{Les acteurs privés: des investissements ciblés et un rôle encore limité dans le renouvellement urbain}

Les entreprises représentent l'un des acteurs du renouvellement urbain et plus précisément de la transformation des anciennes zones de production, qui marquent encore fortement le paysage urbain (carte $n^{\circ} 3$ ). 
Carte 3 : Espaces économiques en mutation dans la ville de Timisoara

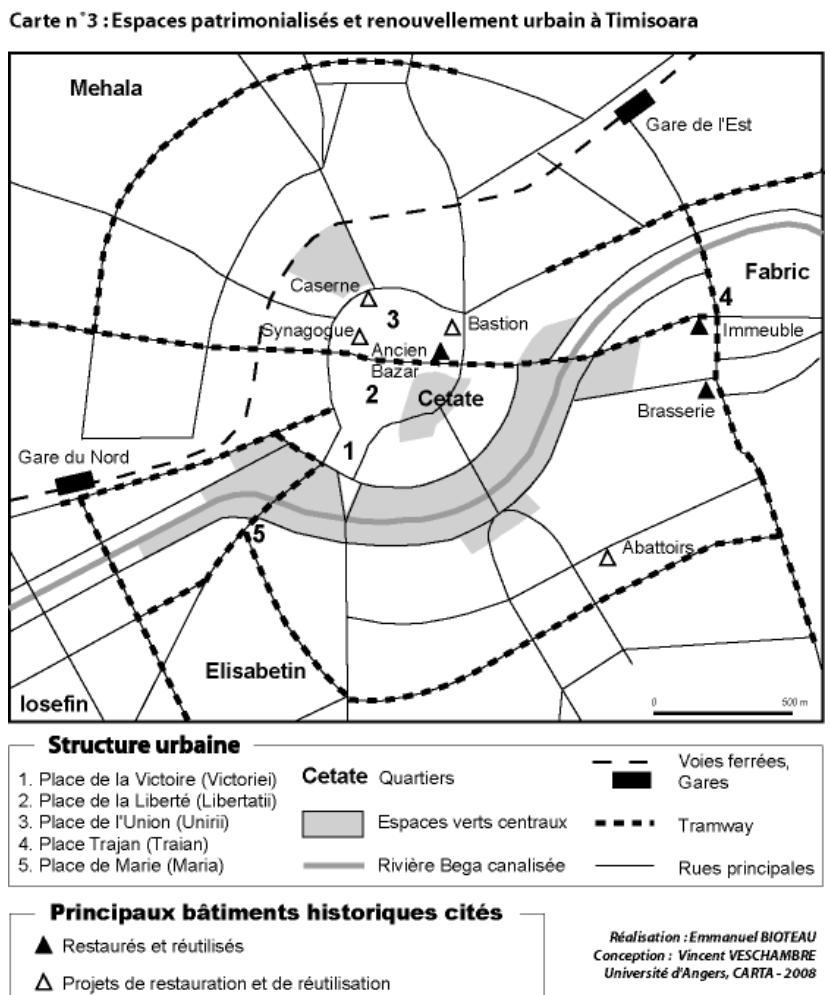

\section{A. Acteurs industriels : des espaces de production aux espaces de mise en scène}

Dans leur ensemble, les zones industrielles de Timisoara (antérieures ou postérieures à la seconde guerre mondiale) n'ont pas été affectées radicalement par la restructuration et la reconversion économique, même si elles ont évolué: le processus de renouvellement urbain tel qu'il s'opère à l'Ouest, dans des espaces anciennement industrialisés, reste donc limité. La tendance générale a été à la réduction de l'activité économique, à la régression de l'équipement technologique, à la conversion vers des activités moins sophistiquées, plus fragmentées, sans envergure nationale, et a fortiori internationale.

En fonction de leur localisation, les anciennes emprises industrielles sont plus ou moins concernées par les investissements et par les évolutions fonctionnelles et paysagères.

En périphérie, les zones industrielles urbaines conservent leurs principales orientations fonctionnelles. C'est le cas de la zone industrielle Calea Buziasului - rue Pestalozzi , la plus étendue et la plus homogène. Celle-ci comprenait de nombreuses unités de l'industrie mécanique, électrotechnique et chimique, du cuir, du textile et de l'agroalimentaire. Certaines entreprises se sont maintenues, reprises par des investisseurs étrangers, comme l'entreprise de détergents rachetée par le groupe américain Procter and Gamble, l'entreprise d'appareils électriques et de mesure, qui forme maintenant le groupe AEM-Luxten ou la fabrique de bière Timisoreana, absorbée par un groupe industriel sud-africain. D'autres usines ont fermé (l'usine d'automobiles Optica, l'entreprise textile Garofita...) ou ont beaucoup réduit leur activité (Technometal, la fabrique de chaussures Guban, Electrotimis ...). Sur des espaces restés libres se sont 
implantées des unités nouvelles, telle que la firme allemande Lisa Drexlmeier, spécialisée dans la production de sous-ensembles et de câbles pour les automobiles, de même que les nouvelles unités de production de Siemens VDO et IBM Roumanie. Les unités reprises ou construites par les compagnies étrangères se démarquent par leurs lignes architecturales modernes et par des aménagements paysagers, qui font contraste avec les terrains restés en friches des anciennes entreprises ou avec ceux, insalubres et dégradés, de certaines firmes locales.

Les plateformes industrielles marquées par la monoactivité, sont inégalement transformées. Le site des Usines Mécaniques de Timisoara au nord-est de la ville a été repris par la compagnie allemande Continental pour la production de pneumatiques. Cette compagnie a démoli presque tous les anciens bâtiments, a construit des halles de production nouvelles et a créé un complexe industriel moderne. Les matériaux utilisés pour la construction des bâtiments, l'aménagement d'espaces verts et de voies d'accès soignées, le choix de la qualité, de l'esthétique et de la propreté ont fait de cette unité un des premiers modèles d'aménagement moderne d'un espace industriel à Timisoara (photo 1). Ce modèle a été repris par des compagnies roumaines prospères, telle qu'Electrica Banat. En revanche, l'activité a cessé sur la plateforme Solventul qui est affectée par une dégradation rapide, même si l'appareil productif était viable, voire performant.

Photo 1 : Nouveaux locaux de la compagnie allemande Continental à Timisoara, situés hors la ville, sur la zone industrielle Calea Buziasului (SE). Ces locaux étaient anciennement propriété de Siemens Automotive, absorbé par Continental en 2008.

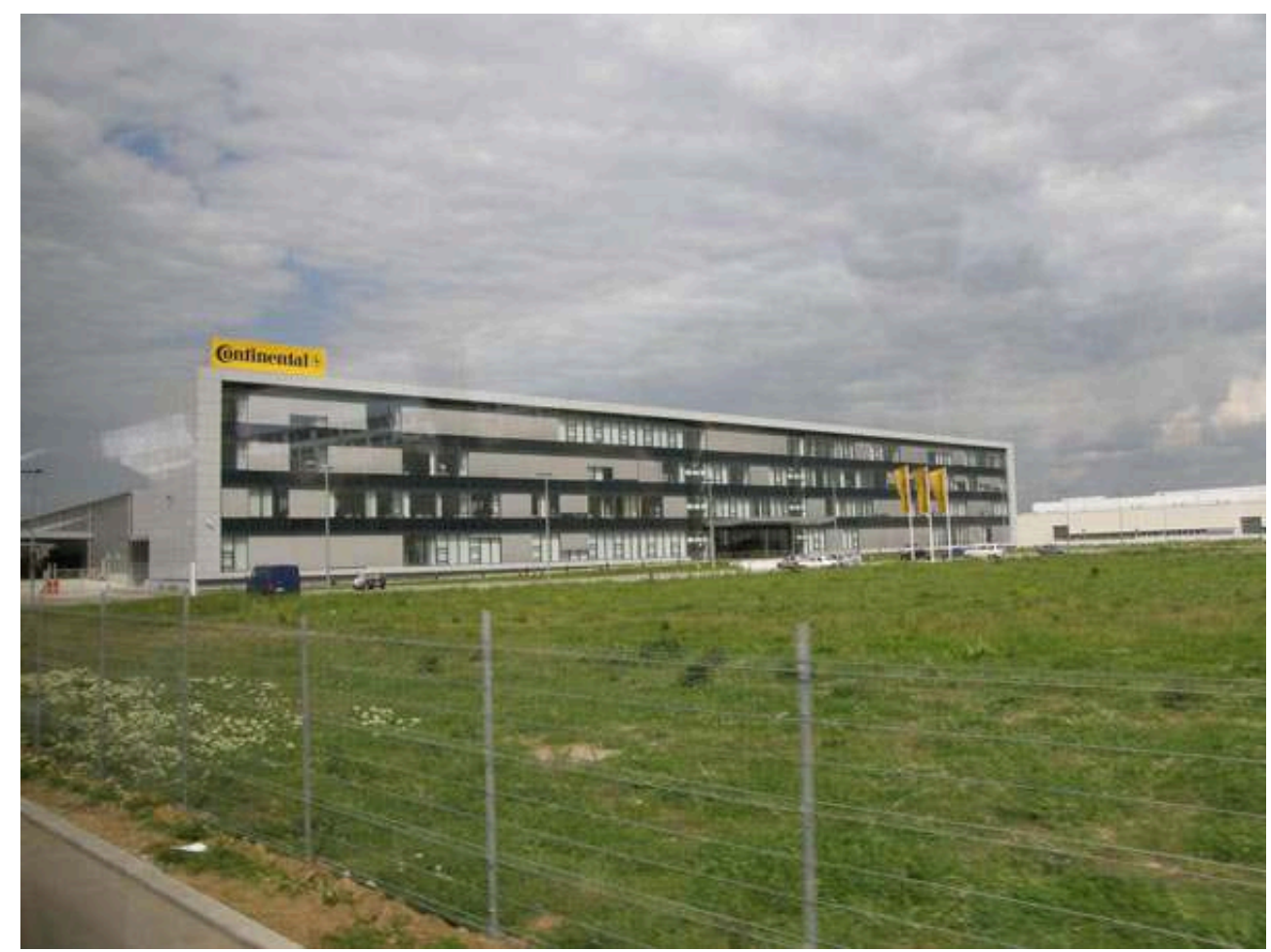

Photo : V.Veschambre, mai 2008

Même si elles ne sont pas toutes concernées, les unités industrielles dispersées dans les espaces centraux et péricentraux sont logiquement les plus attractives, évoluant vers des activités commerciales (photo 2). 
Photo 2 : Exemple de friche industrielle ("Statia de betoane ») située au nord de la Cité, avec en arrière plan une implantation commerciale spécialisée, Praktiker. Elle fait partie de l'ancienne zone industrielle située le long de la voie ferrée, en cours de reconversion (commerces, industries légères et électronique).

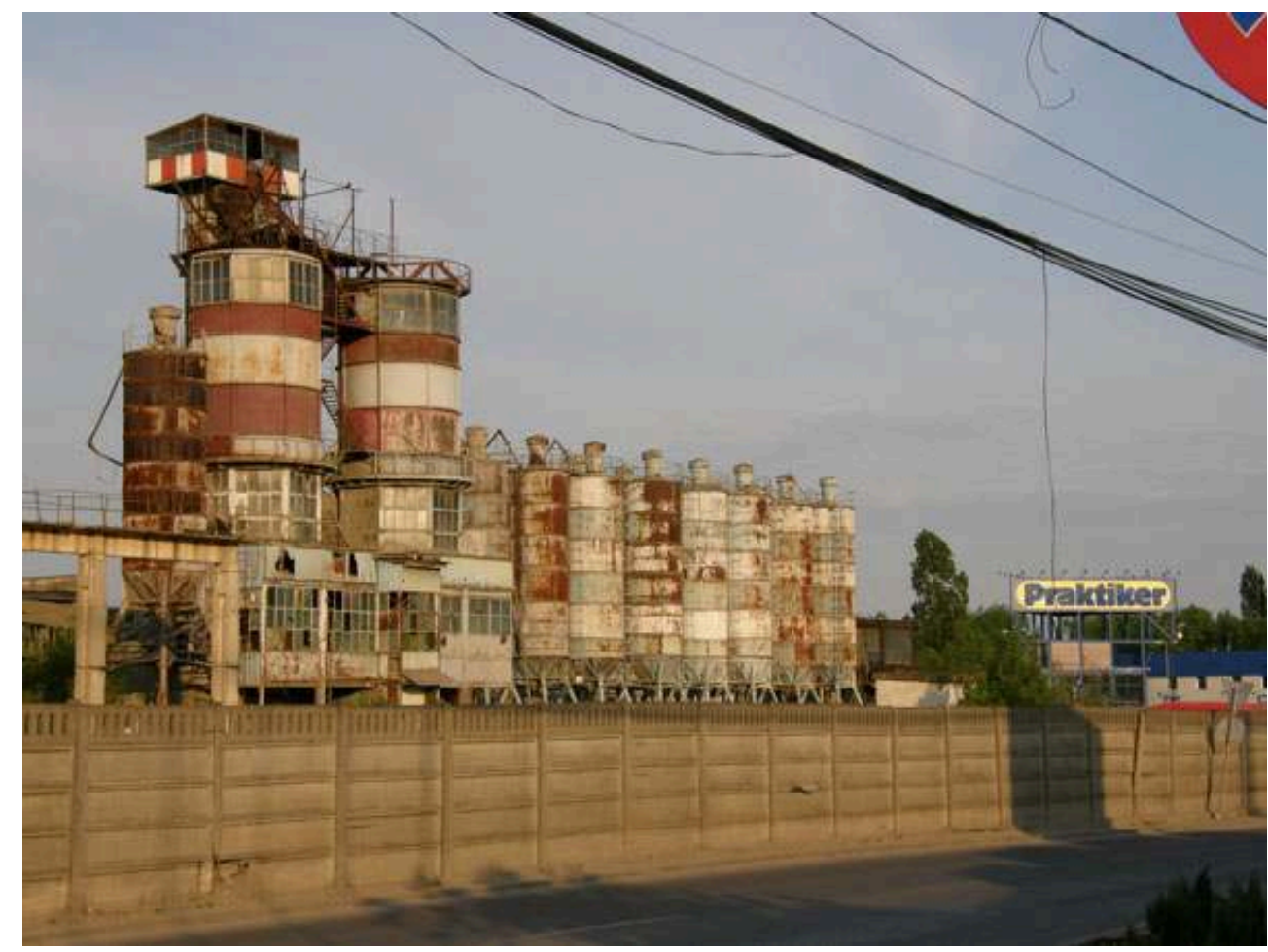

Photo : V.Veschambre, mai 2008

19 Les anciennes unités de production agroalimentaire (Begapam, la Fabrique de lait), les unités de matériaux de construction (préfabriqués de béton), les anciens entrepôts des entreprises commerciales de gros de la période socialiste ont été soit intégralement démolis et remplacés par de grandes unités modernes, principalement commerciales, à capitaux étrangers et roumains, soit ont été repris et remodelés par des groupes roumains qui les ont transformés en supermarchés (Terra, Euro, Kapa).

De grandes compagnies étrangères spécialisées en IT et software se sont installées en position centrale (Alcatel) et péricentrale (Siemens VDO), en reprenant les espaces d'unités de recherches et de production préexistantes (Datatim, Institut de Recherches Hydrotechniques).

21 Les grandes compagnies nationales récemment privatisées, principalement dans le domaine des ressources énergétiques, comme Petrom, Electrica Banat ou la filiale Timis de la Distrigaz Nord, ont fait construire des sièges administratifs modernes, en béton, acier et verre, en localisation péricentrale ou même centrale, symboles de leur puissance économique et financière, contribuant ainsi au renouvellement du paysage urbain. Le siège de la compagnie Petrom se situe ainsi dans l'hypercentre de Timisoara, à proximité des vestiges des anciens remparts (zone Bastion) (photo 3). 
Photo 3 : Implanté dans la ville intra muros, le siège de la compagnie Petrom jouxte le Bastion.

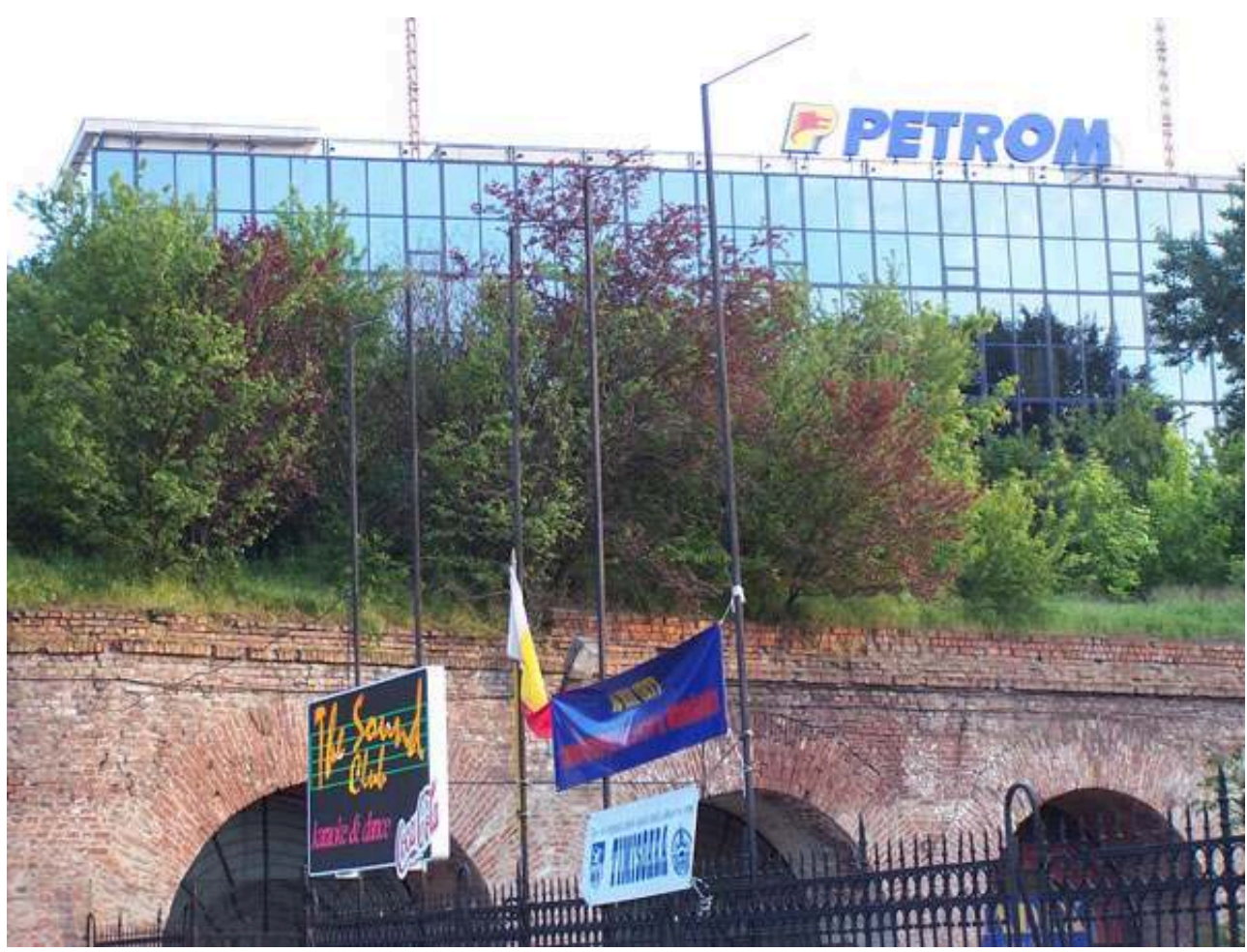

Photo : V.Veschambre, mai 2004

\section{B. Entreprises de services : un impact encore limité sur le renouvellement urbain}

Les espaces destinés aux services ont connu logiquement les mutations les plus significatives. Timisoara a vu son rôle de centre régional se consolider, par la multiplication des centres d'affaires, des lieux d'expositions, des hôtels, des salles de conférences... Par leur architecture moderne, ou à travers la restauration d'anciens équipements (hôtel Continental, Central, Banatul...), ces équipements contribuent à la transformation ponctuelle du paysage urbain, dont s'enorgueillissent les brochures touristiques.

Le secteur bancaire devrait être logiquement le principal levier du processus de développement économique et par conséquent, du renouvellement urbain. Même s'il est bien représenté par plus de 40 succursales et filiales locales des plus grandes banques roumaines et de quelques grandes banques étrangères, le secteur bancaire est encore peu impliqué et insuffisamment organisé pour soutenir le développement local. Les sièges luxueux de ces banques (Banque Roumaine de Développement, Groupe Société Générale, Banque Commerciale Roumaine...), restaurés ou construits pour l'occasion, contribuent certes à la revalorisation du paysage urbain. Cependant, la ville souffre de l'absence d'institutions bancaires locales, en état de promouvoir et de soutenir les intérêts des petits et moyens investisseurs, afin de dépasser les seules réalisations de prestige pour favoriser les investissements locaux et entraîner la généralisation du processus de renouvellement urbain. 

d'urbanité. A Timisoara, on enregistre une forte dynamique commerciale, étroitement liée à la croissance des revenus de ses habitants et au renforcement de ses fonctions de polarisation régionale. magasins, situés en périphérie ou dans les interstices urbains, tels que les magasins Terra, Euro et Kapa, déjà cités, qui ont constitué le premier véritable quartier commercial en dehors du centre ville. A la fin de l'année 2005 s'est ajouté à cette offre le complexe Iulius Mall ${ }^{5}$ Timisoara, à capitaux roumains, situé en position péricentrale, dans la partie nord-est du quartier Cetate, sur une superficie totale de 12 hectares : il s'agissait d'un ancien terrain du Ministère de l'intérieur occupé par des antennes de communication.

La multiplication de ces grands magasins a des effets directs sur les petits commerces des quartiers historiques. Une des préoccupations constantes des autorités publiques locales est de maintenir et de moderniser la présence commerciale dans ces quartiers, jugée indispensable à leur revitalisation. Ces quartiers historiques représentent en effet un enjeu majeur en terme d'image et de dynamique économique.

\section{Le rôle des pouvoir publics locaux : moderniser les infrastructures de transport et revitaliser le centre-ville}

«Afin de contribuer au développement de la ville et améliorer son image, (...) il fut décidé de redonner vie au sentiment de liberté de ses habitants par la « reconstruction » du centre-ville, historique ou nouvellement créé, avec des zones piétonnes commerciales, supermarchés, parcs... ${ }^{6} »$.

Cette idée de « reconstruction» montre que le centre historique constitue un objectif prioritaire pour la municipalité en matière de renouvellement urbain. Partant du principe que ce centre hérité représente un atout important à l'heure où le patrimoine constitue un vecteur d'image important dans la compétition entre villes, la municipalité a cherché à jouer des quelques leviers dont elle dispose. La modernisation du réseau de tramway constitue l'un des vecteurs de renouvellement urbain utilisés par les pouvoirs publics.

\section{A. Miser sur le tramway}

Les efforts d'investissements de la Mairie et du Conseil local de Timisoara se sont tout d'abord orientés vers l'amélioration des infrastructures de circulation et vers l'aménagement du domaine public, spécialement des espaces verts. C'est ainsi que le choix a été fait de réhabiliter les lignes de tramway. L'étalement de la ville (sur une superficie de $130 \mathrm{~km}^{2}$ ), la nécessité de relier des quartiers résidentiels disposés sous forme d'archipel, l'ampleur des flux quotidiens, ainsi que la volonté de revaloriser les trois quartiers historiques ont justifié cette option, prise dans la première moitié des années 1990. La décision a été influencée par l'expérience des grandes villes occidentales qui ont réintroduit le tramway à partir des années 1980 pour limiter l'étalement urbain et la pollution. La présence du tramway est également un vecteur d'image : il est devenu pour les villes qui en sont dotées un symbole de dynamisme et de modernité. A Timisoara, cette question symbolique joue un rôle tout particulier, les 
autorités locales ayant voulu revaloriser et réactualiser un moyen de transport pour laquelle la ville a joué un rôle pionnier: c'est ici en effet qu'a circulé le premier tramway électrique d'Europe, mis en service en 1899, après la création, dès 1867, de la première société de tramways à traction animale.

Compte tenu du degré d'usure avancé du réseau hérité, les responsables locaux ont décidé la reconstruction intégrale des lignes de tramway considérées comme viables économiquement. Les travaux ont commencé simultanément sur plusieurs tracés ${ }^{7}$ et ont avancé à un rythme lent, ce qui a généré une saturation du trafic, dans une période de croissance rapide du parc automobile. Les ressources financières mobilisées par ces travaux sont impressionnantes pour une ville roumaine. Elles s'appuient sur un crédit de 40 millions d'euro obtenus de la BERD et sur le budget local. En conséquence, les fonds restés disponibles pour la réalisation des autres travaux d'infrastructure sont modestes, ce qui se traduit par la dégradation de la voirie routière et par l'aspect insuffisamment soigné des espaces verts, surtout dans les quartiers de grands ensembles. Avec l'étalement des travaux, la ville apparait de plus en plus poussiéreuse, sale et embouteillée, ce qui va à l'encontre des objectifs d'amélioration du paysage urbain et de l'image de la ville. Mais l'on assiste progressivement à l'amélioration du cadre de vie dans les quartiers traversés, au fur et à mesure de l'achèvement des travaux (cf. Carte ${ }^{\circ} 2$ ). D'ores et déjà, le passage du tramway est l'occasion de redessiner les grandes voies empruntées. C'est ainsi que l'axe du boulevard du quartier Josefin a été repris, avec l'implantation d'un nouveau mobilier urbain (photo 4).

Photo 4 : Vue de l'axe principal du quartier Josefin, requalifié par le passage du tramway.

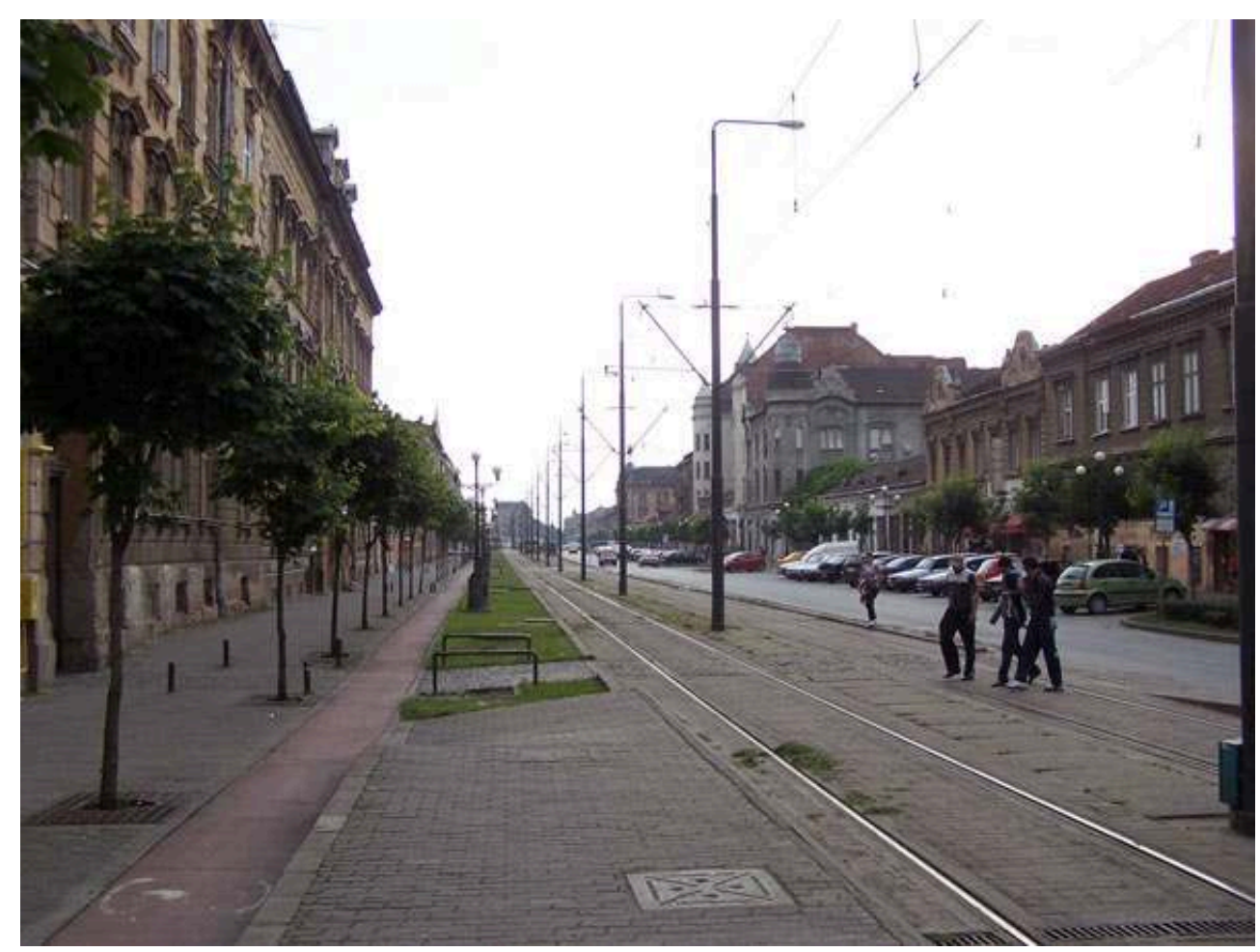

Photo : V.Veschambre, mai 2007

Les conséquences de la modernisation du tramway sur le marché foncier se font sentir. Dès 2004, le message lancé par la puissance publique dans ce quartier avait été entendu par les investisseurs: le nombre de logements, créés dans de grands immeubles du 
début du XXème siècle avait augmenté de $30 \%$, ce qui s'accompagnait d'une augmentation des prix de l'immobilier, qui s'est poursuivie depuis. Mais les responsables municipaux s'avèrent déçus sur deux plans: les commerces n'ont pas connu d'amélioration qualitative ${ }^{8}$ et ce sont surtout les intérieurs des immeubles qui ont été restaurés et non les façades. Quant à l'impact sur l'autre quartier historique de faubourg, le quartier Fabric, il reste en deçà des espérances.

\section{B. Revitaliser les quartiers historiques : ambiance commerciale et valorisation des espaces publics}

31 Si l'on parle à Timisoara de «reconstruction»/revitalisation du centre historique (Cetate, Josefin, Fabric) c'est qu'il y avait du point de vue des autorités locales tout à refaire en matière de paysage urbain, d'ambiance, en un mot, en matière d'urbanité. En d'autres termes, il s'agit dans une logique d'adaptation à l'économie capitaliste contemporaine, de «valoriser » ces espaces et de favoriser leur «consommation ». La priorité a été de promouvoir une présence commerciale modernisée et favorable au développement d'espaces récréatifs, dans un cadre historique valorisant. Même si nous avons vu que cette présence commerciale était soumise à une forte concurrence des grandes surfaces péricentrales, les résultats obtenus ces dernières années sont indéniables. A partir de 2000, les propriétaires ont été incités à louer leurs sous-sols pour implanter des bars, clubs, qui se sont en effet multipliés depuis, notamment dans la Cité. Dans le même temps, l'arrivée d'une nouvelle génération de commerçants a entraîné la modernisation progressive des enseignes du centre ville. La mairie a également fortement incité les cafetiers à investir l'espace public en implantant des terrasses. Entre 2003 et 2006, nous avons constaté l'augmentation du nombre de terrasses et leur extension, sur les principales places du centre-ville ${ }^{9}$. Afin de revaloriser les espaces centraux et contribuer à leur marchandisation, la mairie organise également des évènements festifs, pour attirer notamment une population jeune avec des concerts gratuits ${ }^{10}$ (photo 5 ). 
Photo 5 : Place de l'Union (Unirii), dominée par la cathédrale romano-catholique et investie par les terrasses de cafés et les scènes de concerts.

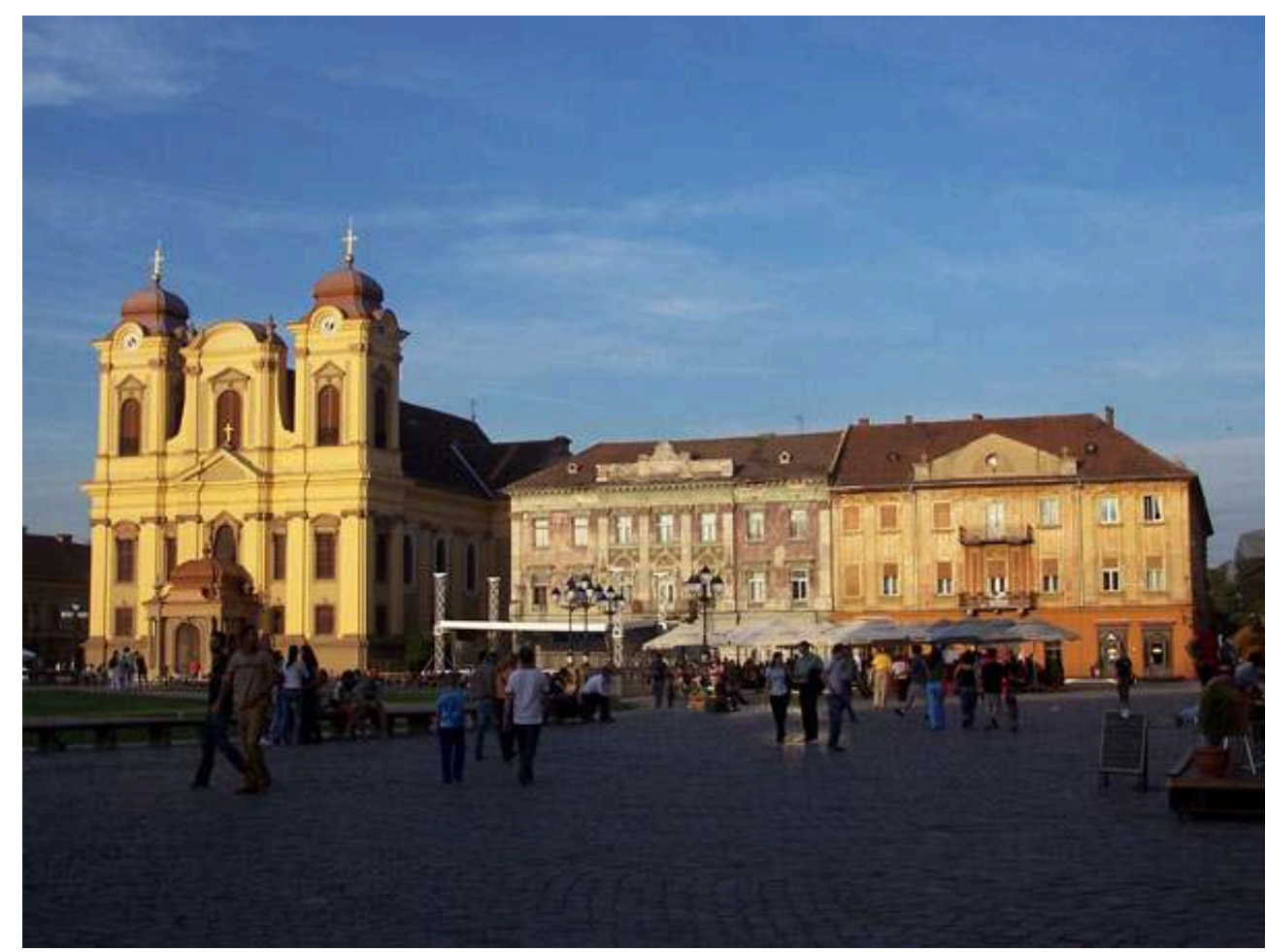

Photo : V.Veschambre, mai 2007.

Cette population jeune, notamment étudiante, constitue selon les responsables des services de l'urbanisme une clientèle particulièrement stratégique pour revitaliser le centre historique de Timisoara. Après 1989, la vie urbaine la plus dynamique - surtout la vie nocturne, avec son atmosphère typiquement urbaine - s'est concentrée petit à petit dans le campus universitaire (Complexul studentesc), qui s'est ainsi transformé en un véritable quartier de distractions, cosmopolite et bruyant, ce qui n'est pas sa fonction a priori. L'objectif est donc de réorienter cette animation au profit des quartiers historiques. Pour cela, les autorités locales tablent sur la capacité des universités à jouer un rôle majeur en matière de renouvellement urbain. En effet, la croissance extraordinaire du nombre d'étudiants après 1989 a déterminé les universités de Timisoara, surtout l'Université de l'Ouest, à faire des acquisitions immobilières pour étendre les espaces destinés aux activités didactiques et de recherches. Ont été récupérés ainsi certains bâtiments qui avaient appartenu aux universités les décennies précédentes, tandis que d'autres immeubles étaient rachetés et réutilisés ${ }^{11}$. Parmi ces transformations, il faut citer notamment celle de l'ancienne mairie située en plein centre-ville, occupée partiellement par la faculté de musique et celle d'une ancienne caserne $^{12}$ située immédiatement au nord de la Cité, devenue faculté des arts. Se sont constitués ainsi plusieurs espaces universitaires: le noyau initial, qui reste le plus vaste, situé au sud de la Cité, puis le noyau central de la place de la Liberté, et enfin celui situé en bordure nord-est de la Cité. A travers sa reconversion et son remodelage, cet ancien ensemble militaire a vocation à devenir le deuxième campus universitaire de la ville. Dans l'esprit des responsables locaux, le flux de jeunes circulant entre les différents campus, à travers le centre-ville, devrait contribuer à sa revitalisation. Une 
réflexion équivalente est menée à propos du quartier Fabric, où l'ancienne brasserie Timisoreana (devenue lieu de restauration) constitue un pôle d'attraction.

Afin de promouvoir ces activités commerciales, festives, les autorités tentent d'agir sur la revalorisation des espaces publics, les places notamment, qui constituent le cadre privilégié de ces animations. Ces places constituent des éléments emblématiques de la ville, contribuent fortement à l'image de marque positive dont elle bénéficie et représentent une forte valeur symbolique pour les habitants. Du point de vue historique et patrimonial, la place de l'Union (Piata Unirii) constitue l'héritage le plus ancien et le plus important de la ville (photo 5). De forme carrée, la place est entourée par l'un des ensembles architecturaux les plus prestigieux de Roumanie, et constitue un modèle de place baroque à l'échelle centre-européenne. Restée longtemps en marge du centre-ville, cette place fait depuis quelques années l'objet d'une attention spéciale de la part des autorités. La restauration des immeubles les plus significatifs qui la bordent (cathédrale romano-catholique, cathédrale orthodoxe serbe, Musée d'art...), l'augmentation du nombre des événements culturels qui s'y déroulent et surtout, la multiplication des locaux commerciaux de standing et des terrasses ont réussi faire de la Place de l'Union un lieu attractif.

Mais c'est avec la place de la Victoire (Piata Victoriei) que la charge symbolique est la plus forte pour la population de Timisoara. Vaste quadrilatère dominé principalement par des immeubles de l'époque 1900, la place est orientée vers les deux principaux symboles de Timisoara: la cathédrale orthodoxe roumaine et le bâtiment de l'Opéra national et du Théâtre national de Timisoara (photo 6).

Photo 6 : Place de la Victoire avec la statue de la louve, symbole de la romanité et l'opéra en arrière plan, haut lieu de la Révolution roumaine.

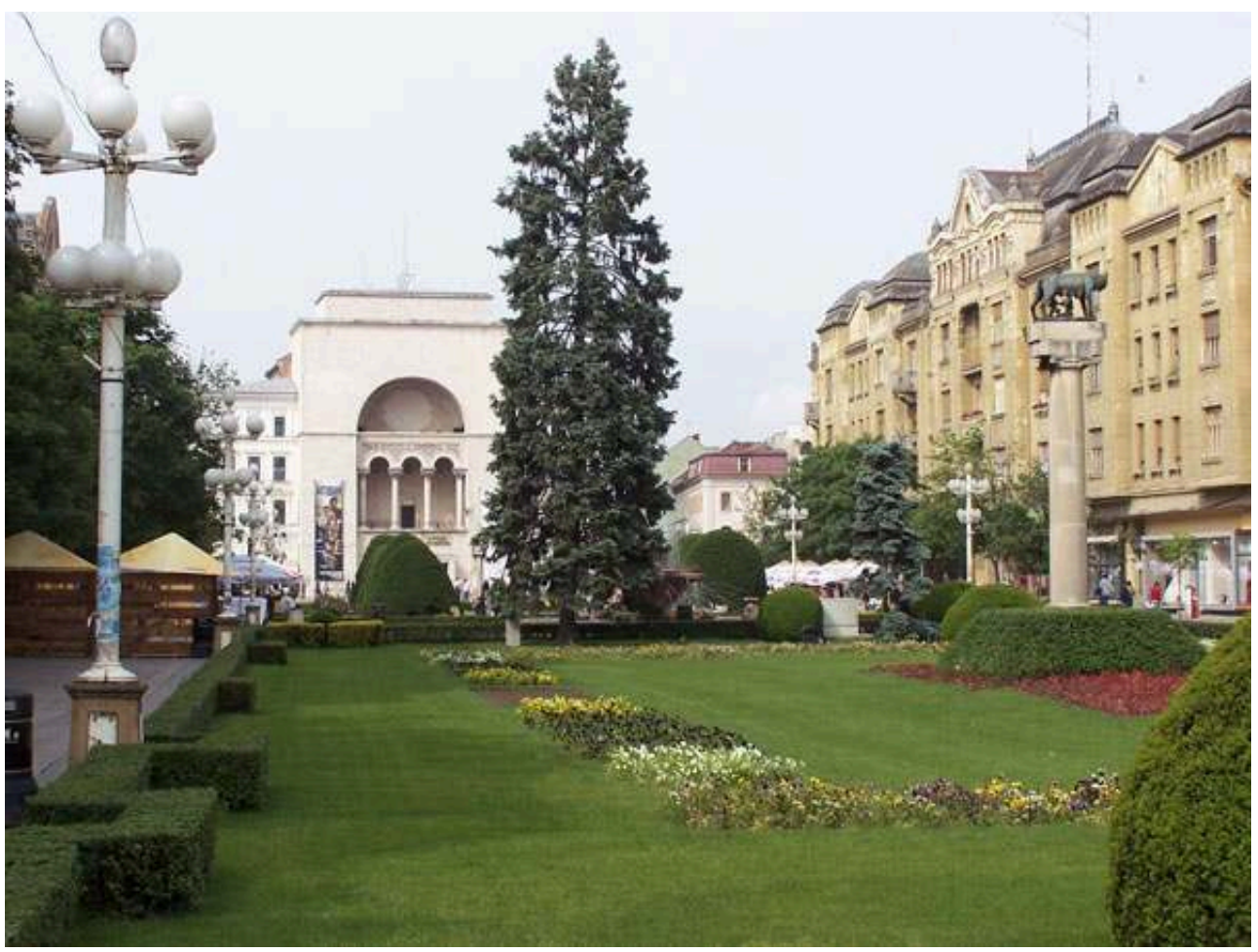

Photo : V.Veschambre, mai 2008 
Du point de vue urbain, la Place de la Victoire a été parachevée à la fin des années 1980, peu avant la chute du régime communiste. Les lignes de tramway ont alors été déviées et la place a été consacrée entièrement à la circulation piétonnière et aux espaces verts. Peu après cette place est devenue le symbole de la résistance anticommuniste et de la lutte pour la liberté : c'est ici que se sont réunis des dizaines de milliers d'hommes et de femmes de Timisoara, à partir du 16 décembre 1989, pour protester contre la dictature. Du balcon de l'Opéra, Timisoara a été proclamée «ville libre du communisme », la première de Roumanie. Sa position de couloir entre de grands boulevards et des rues piétonnières intensément pratiquées, la proximité des principales infrastructures socioculturelles et commerciales, dans le voisinage des plus beaux parcs de la ville, accentuent la fonction polarisatrice de la place.

Si elles ont connu une revitalisation commerciale, les deux places principales de la ville n'ont pas fait l'objet de rénovations d'envergure et ont finalement conservé l'aspect hérité de la période communiste ${ }^{13}$. En revanche, les places des quartiers historiques péricentraux ont fait l'objet d'interventions urbanistiques, afin de renforcer leur attractivité. C'est le cas de la place Trajan du quartier Fabric, important nœud de transport urbain, dont la structure et la surface ont été entièrement reprises. (photo 7)

Photo 7 : Place Trajan, redessinée par le passage du tramway. En arrière plan des immeubles jugenstil en attente de réhabilitation.

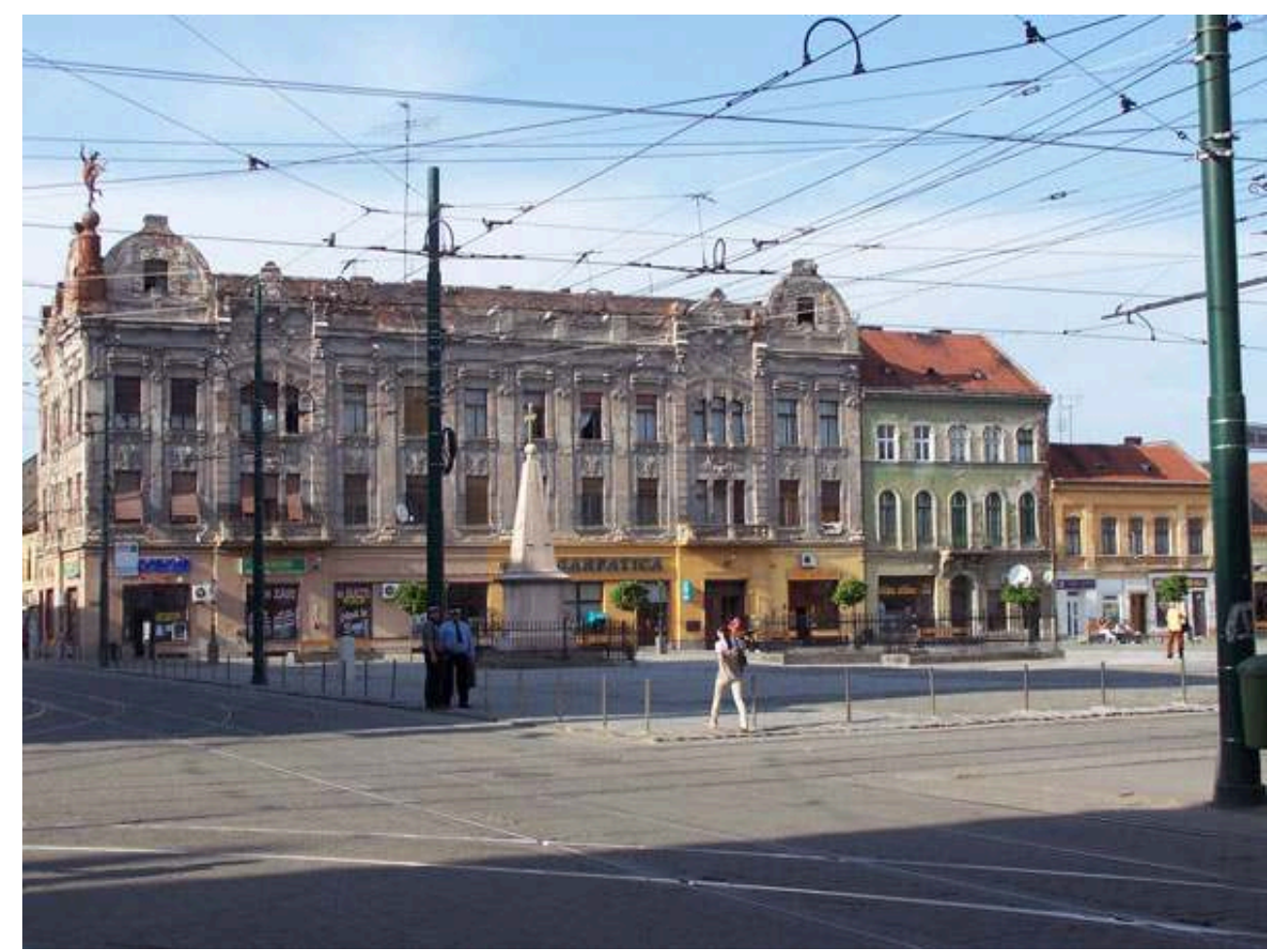

Photo : V.Veschambre, mai 2008

Au-delà de ces espaces stratégiques pour l'image de la ville, les autorités locales tentent d'impulser plus largement un processus de réhabilitation du patrimoine urbain dans son ensemble. 


\section{Patrimoine et renouvellement urbain à Timisoara}

La question de la revitalisation du centre-ville est bien entendu intimement liée à celle de la mise en valeur du patrimoine. Depuis quelques décennies, le patrimoine a pris une importance grandissante dans l'imagerie urbaine, en Europe notamment. Et cette problématique du patrimoine interfère nécessairement avec celle du renouvellement urbain, qui doit tenir compte de l'héritage prestigieux des quartiers historiques. La ville de Timisoara n'ayant pas été touchée par le processus de systématisation des années 1970-80 $0^{14}$, elle dispose d'un atout important en la matière dans le contexte roumain (Popa, 2000) : ce sont 91 éléments (70 éléments ponctuels et 21 ensembles ou sites urbains) qui sont ainsi protégés au titre des monuments historiques et 14000 immeubles qui sont considérés comme "historiques" par la mairie. Mais selon une enquête réalisée en 2005 pour la mairie, 11\% seulement de ces immeubles ont été restaurés partiellement ou totalement, la moitié seulement de ces restaurations ayant été respectueuses du bâti ancien.

Les autorités locales ont conscience que laisser ce patrimoine dans l'état de délabrement dans lequel il se trouve pourrait à terme s'avérer au contraire dissuasif pour les investisseurs. Les trois principaux quartiers historiques (Cetate, Josefin, Fabric), désormais protégés dans le cadre d'un zonage ${ }^{15}$, font l'objet de la plus grande attention.

\section{A. Un patrimoine reconnu encore insuffisamment mis en valeur}

Même s'il était reconnu et officiellement protégé ${ }^{16}$, le patrimoine architectural de la ville n'a pas été entretenu durant la période communiste. Comme cela a généralement été le cas dans les villes des pays anciennement communistes, les investissements ont privilégié le logement collectif de la périphérie. Par ailleurs, le morcellement des appartements dans un contexte de grave crise du logement a accentué les phénomènes de dégradation.

Une loi de 1991 a contraint la puissance publique à vendre les logements aux anciens occupants. La vente s'est faite en dehors des prix du marché, à des habitants plutôt pauvres et âgés et la puissance publique a perdu l'essentiel de ses capacités d'intervention. Si certains ont revendu dans une logique spéculative, et si l'on assiste à une amorce de gentrification (Cetate, Josefin), le niveau de vie moyen reste faible et le morcellement des immeubles très prononcé. Les parties communes de ces immeubles, dont les façades, ne sont pas entretenues car les copropriétaires n'en ont pas les moyens. Plus grave, les structures de nombreux immeubles sont menacées par l'humidité du sous-sol et le manque d'entretien des charpentes.

Même si la ville possède encore 6000 logements dispersés dans la ville, elle ne peut guère intervenir. La loi sur les monuments historiques impose la protection des immeubles protégés ou localisés dans les zones historiques (zone istorice urbane), mais la loi financière interdit l'usage de moyens publics pour l'entretien d'une propriété privée. Donc si la mairie possède un ou plusieurs appartements dans un immeuble historique elle ne peut intervenir à l'échelle de l'immeuble tout entier. Elle peut agir dans les rares cas où elle est seule propriétaire. C'est ce qui s'est produit pour un immeuble de la place Trajan dans le quartier Fabric: les fondations ont été renforcées et l'immeuble réhabilité de manière soignée (carte $\left.n^{\circ} 2\right)$. Ce type de restauration reste 
encore trop ponctuel pour avoir un impact sur l'évolution urbaine. Mais elle a valeur d'exemple : c'est ainsi que plusieurs immeubles résidentiels ont été restaurés par des privés ou sont en cours de travaux dans le secteur de la Place de l'Union.

Compte tenu de ses faibles moyens d'action, que ce soit du point de vue juridique ou financier, la mairie se tourne vers des partenaires étrangers. C'est ainsi qu'elle s'est attaché les services de l'organisme allemand GTZ, financé par le Ministère fédéral pour la coopération et le développement ${ }^{17}$. Dans le cadre du programme "réhabilitation respectueuse et revitalisation économique des quartiers historiques» (2006-2008), cet organisme propose des diagnostics, des formations et des conseils (restauration, financement) gratuits aux propriétaires et artisans concernés. Il contribue également à la sensibilisation du public.

Par ailleurs, des financements allemands ou européens ont été obtenus par la mairie (statuaire baroque, Bastion) ou sont en cours de négociation (Places de l'Union, de la Victoire) pour certaines restaurations de prestige.

\section{B. Patrimoine et recyclage : les enjeux du renouvellement urbain}

Comme dans tout processus de renouvellement urbain, la tentation de la démolition existe, compte tenu de la pression exercée par le marché immobilier sur les prix du foncier dans les quartiers centraux. Nombreux sont les acquéreurs potentiels de bâtiments anciens qui ne sont pas intéressés par les immeubles en tant que tels mais par les surfaces qu'ils occupent, dans la perspective de les remplacer par des constructions neuves beaucoup plus rentables, certains attendant que leur immeuble finisse par s'écrouler ${ }^{18}$. Il s'agit pour les autorités locales de résoudre la contradiction potentielle entre la volonté de conserver les héritages bâtis et la volonté d'attirer les investisseurs dans les quartiers anciens.

C'est la question du patrimoine, dans sa relation au renouvellement urbain, qui est ainsi posée. Cette notion de patrimoine est liée à l'obsolescence d'un héritage et à la reconnaissance d'une nouvelle valeur, qui n'est plus celle de la valeur d'usage. Mais pour que le patrimoine soit conservé et revalorisé, cela suppose qu'il soit " habité " (Gravari-Barbas, dir., 2005), c'est à dire réutilisé, généralement avec une fonction différente de sa fonction d'origine. Par rapport aux années 1950-60, où l'intervention sur le tissu urbain hérité passait fréquemment par la table rase, dans les années 1990-2000, ce que l'on a appelé renouvellement urbain s'appuie bien souvent sur la patrimonialisation d'éléments jugés significatifs et valorisants, qui sont ainsi réinvestis et intégrés dans les projets urbains.

La patrimonialisation renvoie donc à une dialectique entre conservation et recyclage qui n'est pas sans soulever des problèmes concrets. Cette question s'est posée à propos des abattoirs de Timisoara. Construit en 1904-1905 (Munteanu, 1995), classé monument historique, cet ensemble architectural se situe au milieu d'un terrain de 4 hectares, au voisinage immédiat du campus universitaire (photo 8). 
Photo 8 : Les abattoirs avant les travaux de transformation en centre commercial.

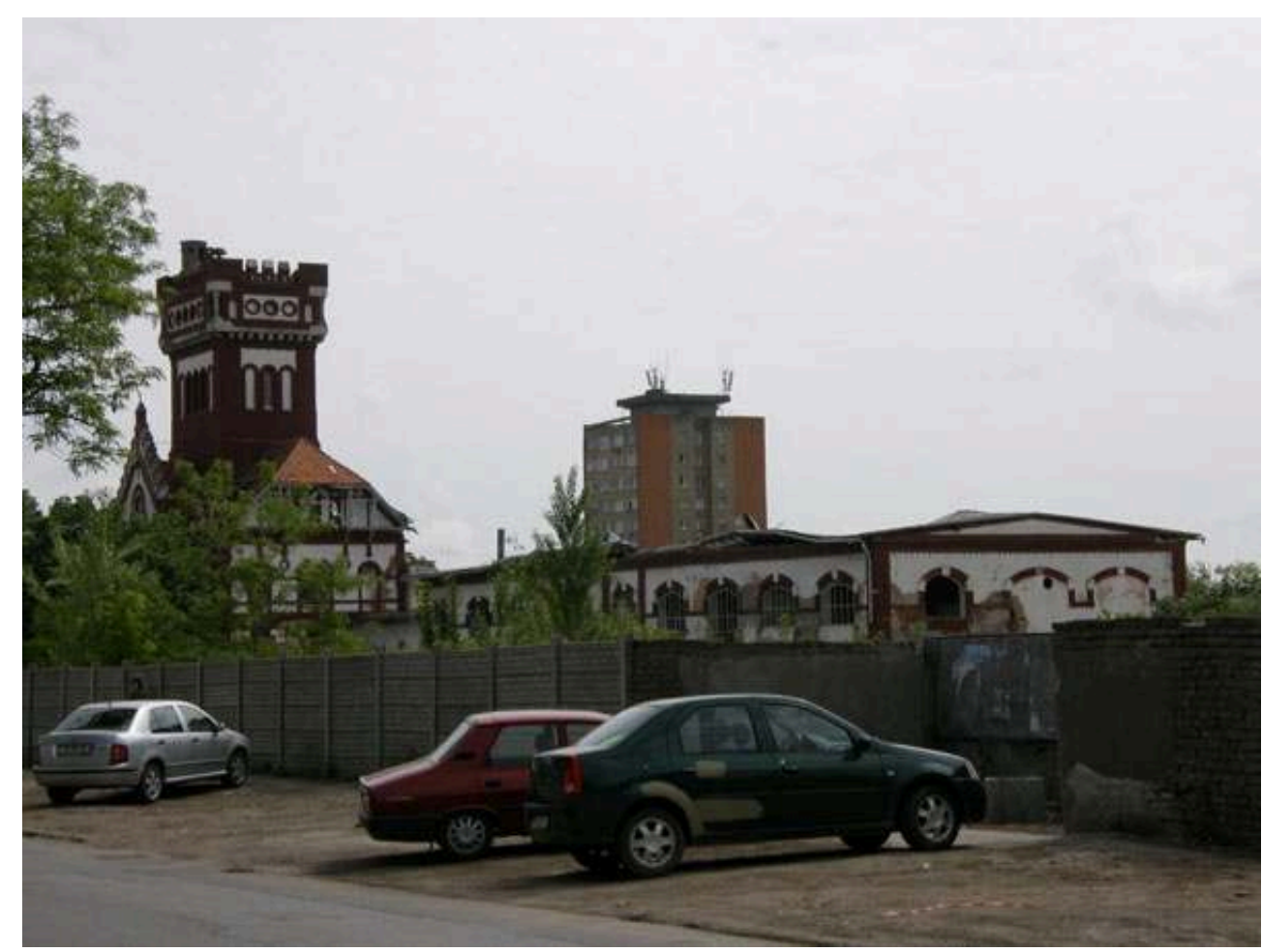

Photo : V.Veschambre, mai 2008

Abandonnés après leur fermeture, les abattoirs ont été rachetés pour être transformés en centre commercial, compte tenu de leur position remarquable. Le projet n'a pas reçu les avis favorables de la commission nationale pour les monuments historiques, qui a jugé que l'aménagement du centre commercial, qui ne conservait que les façades, aurait conduit à une trop forte dénaturation. Selon la mairie, la commission des monuments historiques a fait échouer un projet de développement économique, tandis que pour la commission, la mairie était prête à sacrifier un monument historique pour obtenir cet investissement. Un second projet de centre commercial, plus respectueux, est cependant aujourd'hui en bonne voie.

Une situation similaire a été observée à propos de la Caserne U de la Place Marasesti, ensemble particulièrement vaste situé en plein centre-ville (cartes $n^{\circ} 1$ et 2 ) : le projet de mise en valeur comme centre multimédia (par la firme Média Pro) n'a pas abouti et le bâtiment continue de se dégrader. La mairie est en train d'en faire l'acquisition, ce qui va ouvrir de nouvelles perspectives. Par leur ampleur et leur prestige moindre, ces éléments de patrimoine industriel ${ }^{19}$ ou militaire sont sans doute ceux pour lesquels conservation et recyclage sont les plus difficiles à concilier.

50 Il y a cependant un certain nombre d'expériences réussies et de projets en cours, où l'on réussit à concilier conservation et réutilisation d'édifices emblématiques. $\mathrm{Ne}$ rentrent pas à proprement parler dans cette catégorie les restaurations engagées par des institutions qui n'ont fait que remettre aux normes et embellir leur siège : le Théâtre national de Timisoara ${ }^{20}$, le Musée d'Art (Palais baroque de la Place Unirii), la Poste, la Banque Nationale de Roumanie, l'hôpital municipal ou le Ministère de la Justice (Palais discatérial). 
Plus originale est la mise en valeur des fragments du rempart détruit au début du XXème siècle. Le principal secteur conservé, le Bastion, accueille un musée d'ethnographie, un centre d'expositions et plusieurs espaces commerciaux de loisirs logés dans l'épaisseur de ses murs. Une restauration encore plus ambitieuse a été engagée en 2007 grâce à des crédits européens, afin d'améliorer l'attractivité de ce secteur de la Cité. La restauration récente du palais dit de l'ancien Bazar (carte $\mathrm{n}^{\circ} 2$ )mérite également d'être signalée comme première réhabilitation d'envergure réalisée par un privé. L'ayant revendiqué et gagné en justice, son nouveau propriétaire l'a rénové et loué aux diverses entreprises et institutions intéressées, parmi lesquelles l'Agence de Développement Régionale Ouest, qui dispose ainsi d'un siège à la fois central et valorisant.

52 Enfin, le projet de restauration-réutilisation le plus ambitieux concerne la synagogue de la Cité, bel édifice de style néo-mauresque (photo 9).

Photo 9 : La synagogue de la Cité, en attente de réhabilitation.

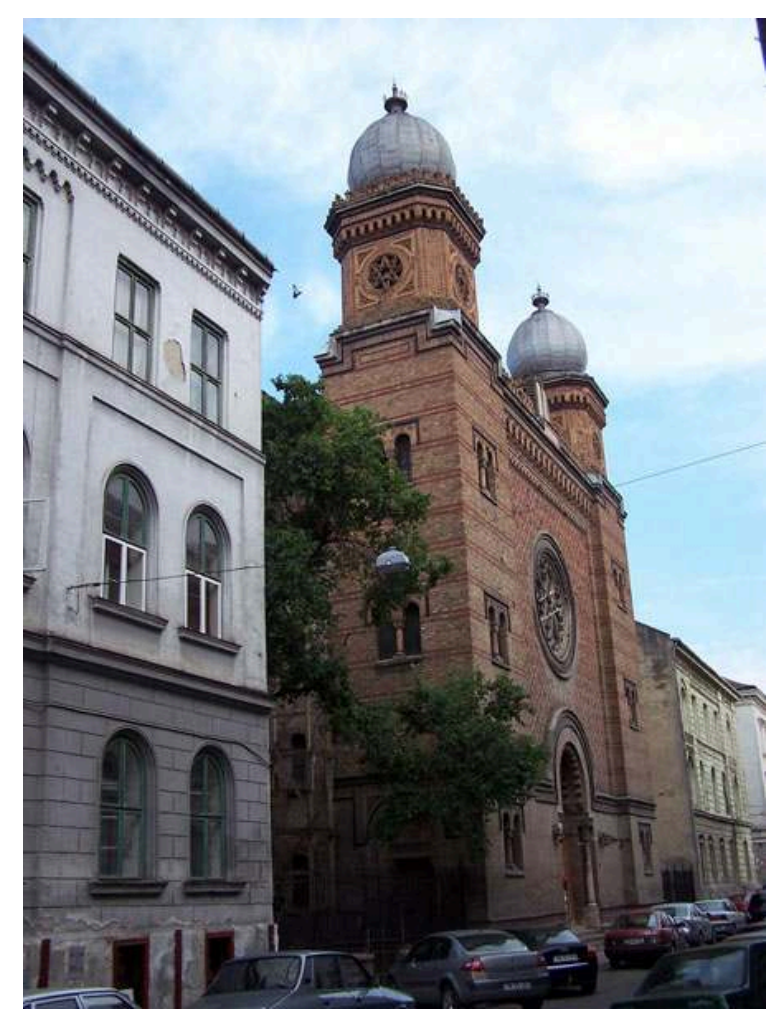

Photo : V.Veschambre, mai 2007

53 A cause des coûts élevés d'entretien des trois synagogues de la ville, la communauté juive a convenu avec les autorités locales de donner cet édifice en concession à la ville, qui veut en faire un espace culturel.

\section{Conclusion}

L'essor économique remarquable, dû surtout aux investissements étrangers, que connaît Timisoara n'entraîne pas un renouvellement urbain aussi important que celui auquel on aurait pu s'attendre. Plusieurs hypothèses peuvent être avancées pour 
expliquer ce décalage. Les entreprises implantées ne génèrent sans doute pas de revenus suffisamment importants, ni pour les individus, ni pour la collectivité. Ensuite, la distribution des ressources publiques (taxes et impôts), trop centralisée, ne laisse pas suffisamment de moyens sur place, pour couvrir les besoins de modernisation de la ville. De plus, l'utilisation des fonds budgétaires de la ville est encore orientée vers des secteurs lourds et peu visibles. A ces raisons économiques s'ajoute le contexte juridique : contrairement à ce qui s'est passé chez le voisin hongrois, la puissance publique s'est privée de leviers d'intervention en aliénant l'essentiel de son parc public. Trop démunis ou peu sensibilisés au patrimoine lorsqu'ils en ont les moyens, les propriétaires sont encore peu nombreux à s'engager dans la mise en valeur de leur patrimoine ancien.

Quand on compare le paysage urbain de Timisoara à celui des villes voisines de Szeged (Hongrie) et même de Novi Sad (Serbie), on ne peut pas éviter de reconnaître un certain retard en matière de mise en valeur du cadre urbain, alors que son économie est la plus dynamique des trois villes. La ville de Timisoara demeure un pôle dans lequel on investit pour jouir du coût de la main d'œuvre (encore) faible, sans que la valeur ajoutée ne s'accumule encore localement sous la forme d'un patrimoine immobilier revalorisé.

\section{BIBLIOGRAPHIE}

Bioteau E. (2005). - Des frontières et des hommes. Approche des recompositions territoriales frontalières : l'exemple de l'Ouest roumain, Thèse de doctorat de Géographie, Angers, Université d'Angers, 418 p.

Dobraca L. (1997). - Les activités commerciales dans les villes roumaines. Etude géographique, thèse de doctorat, Université de Bucarest.

Giurescu D. (1990). - The razing of Romania's past, London, Architecture design and technology press, $69 \mathrm{p}$.

Gravari-Barbas M. (dir.) (2005) - Habiter le patrimoine : enjeux, approches, vécu, Rennes, PUR, 618 p.

Junie A. (2002). - Municipiul Timisoara, Timisoara, éditions Almanahul Banatului, 32 p.

Maggi V. (2002). - Timisoara : 4 passi per la città, Timisoara, Editura orizonturi universitare, 123 p.

Munteanu I. (1995). - Ghidul Municipiului Timişoara, Timisoara, Editions Helicon.

Popa N. (2000). - "Identitate, teritorialitate şi prestigiu cultural în spaŢiul geografic românesc

(I) », in vol. Regional Conference of Geography "Regionalism and integration: culture, space, development", the papers of IVth edition, Timişoara, Angers, Tubingen, p. 87-95.

Popa N. (2004). - « Le rôle des PME dans le développement régional : le cas des investissements italiens dans le Banat roumain ", article présenté au Séminaire international Initiatives entrepreneuriales et développement régional, comparaisons européennes, Potsdam, 16 p.

Popa N., Junie A. (2000). - "The geographical, national and european position of Timişoara City and its perspective of development", in vol. Regional Conference of Geography "Regionalism and 
integration: culture, space, development", the papers of IVth edition, Timişoara, Angers, Tubingen, p. 25-37.

VoiculescuS. (2004). - Oraşele din Câmpia de Vest. Structuri şi funcţionalităŢi urbane, Timişoara, éditions UniversitǎŢii de Vest, 253 p.

\section{NOTES}

1. Entre 1990 et 1999, au registre du commerce de Timisoara ont été enregistrées 4485 sociétés à participation étrangère, auxquelles se sont ajoutées 4317 autres sociétés, entre 2000 et 2007 (Mairie de Timisoara, 2008).

2. Ce n'est pas un hasard si le déclenchement de la Révolution de décembre 1989 a eu lieu dans cette ville.

3. La ville se trouve à moins de $600 \mathrm{~km}$ de 10 capitales européennes.

4. Entretien collectif au service des monuments historiques, préfecture, 20 mai 2004.

5. Signé par le grand architecte roumain Radu Mihailescu.

6. Junie Aurelia, 2002, Municipiul Timisoara, p. 30 (traduction du roumain).

7. Les lignes de tramway en modernisation après 1999 ont une longueur totale de 40,1 km, sur 14 tracés. Deux tiers de ces lignes ont été déjà remis en fonction.

8. La création du mall a sans doute capté les enseignes les plus valorisantes.

9. Dans nos comptages, nous sommes passés de 23 à 29 terrasses entre ces deux dates (soit + 25\%), l'essentiel de la progression concernant la place Unirii (carte $n^{\circ} 1$ ).

10. Signalons le festival Mica Vienna au mois de mai, qui fait référence de manière significative au passé autrichien de la ville et la fête de la bière au mois de juin.

11. Sur le campus lui-même est engagé un programme de réhabilitation des cités universitaires qui sont marquées par la vétusté.

12. Cette problématique du recyclage des anciens bâtiments militaires dans les villes roumaines est particulièrement intéressante.

13. Si l'on excepte bien entendu la construction d'un monument à la Révolution face à la cathédrale orthodoxe roumaine, sur la place de la Victoire.

14. Dans son ouvrage The razing of Romania's past, Dinu Giurescu comptabilise 29 villes rasées à plus de $85 \%$ et 37 villes où les démolitions ont été significatives (Giurescu, 1990).

15. Les zonages de protection ont été instaurés à partir de 1992.

16. La liste des monuments historiques publiée en 1992 reprend très largement celle de 1960 (loi de 1955), ce qui montre une certaine continuité. Mais ce n'est qu'au début des années 1980 que l'intérêt pour les monuments historiques a véritablement commencé à se manifester (cf. aménagements de la Place Unirii).

17. Société allemande pour la coopération technique. Cet organisme a fait ses preuves à Sibiu, capitale européenne de la culture 2007.

18. C'est ainsi que deux immeubles se sont effondrés durant ces dernières années dans la Cité.

19. Timisoara compte 6 éléments de patrimoine industriel protégé, dont un seul, la brasserie Timisoreana, a fait l'objet d'une véritable mise en valeur.

20. Restauration de prestige qui contribue au renforcement de la fonction commerciale de la rue piétonne Alba Iulia 


\section{RÉSUMÉS}

Timisoara est une des villes roumaines privilégiées par sa situation et son histoire, ce qui lui permet d'attirer les investisseurs étrangers. Dans un tel contexte, nous nous sommes demandés si la ville connaissait un rythme de renouvellement urbain particulièrement élevé et si les réalisations urbanistiques étaient à la hauteur du rôle que Timisoara semble être appelée à jouer dans cette partie de l'Europe. Nous apportons des éléments de réponse en envisageant à la fois les interventions des acteurs privés dans les espaces d'activités et le rôle des acteurs publics autour de la mise en valeur des espaces publics et des héritages urbains. Nous proposons donc un tour d'horizon des processus de renouvellement urbain dans une métropole régionale d'une Europe centre-orientale en pleine mutation.

Among the outstanding Romanian cities, Timisoara and its privileged situation and history are potentially attractive to foreign investors. In this context, the question is raised to know if this city is undergoing a fast regeneration process and if the urban planning and achievements are in accordance with the part Timisoara is called to play in this part of Europe. In this paper, we provide a few answers with the analyses of the initiatives of private actors in areas of activities and the role of public actors in the development of public spaces and urban heritage. We also consider from all angles the urban regeneration processes undertaken in this large and changing Eastern European regional centre.

Timisoara ist eine der rumänischen Städte, die durch ihre Lage und ihre Geschichte privilegiert ist. Demzufolge zieht sie ausländische Investoren an. In diesem Kontext haben wir uns gefragt, ob die Stadt einen besonders starken Umwandlungsprozess erlebt und ob die städtebaulichen Möglichkeiten und Umsetzungen der Rolle entsprechen, die Timisoara in diesem Teil Europas spielen soll. Wir versuchen eine Antwort zu finden, indem wir zugleich den Einsatz der privaten Akteure in den Gewerbegebieten und die Rolle der öffentlichen Akteure bei der Erschließung öffentlicher Plätze und des städtischen Erbes untersuchen. Wir wollen damit einen Ausblick auf städtische Erneuerungsprozesse in einer Regionalmetropole im sich wandelnden Zentral-OstEuropa geben.

\section{INDEX}

Schlüsselwörter : lokale Entwicklung, städtische Verbesserung, Kulturgut, Timisoara, Rumänien Mots-clés : développement local, renouvellement urbain, patrimoine, Timisoara, Roumanie Keywords : local development, urban regeneration, heritage, Timisoara, Romania

\section{AUTEURS}

\section{VINCENT VESCHAMBRE}

Département de Géographie - Université Blaise Pascal (Clermont II) - 29, Bd Gergovia - 63037

Clermont-Ferrand - vincent.veschambre@univ-bpclermont.fr

\section{NICOLAE POPA}

Département de Géographie - Université de l'Ouest - Bd.Vasile Pârvan no.4 - RO 300223 -

Timişoara - România - npopa61@yahoo.com 